International Journal of Pure and Applied Mathematics

Volume 86 No. 1 2013, 55-63

ISSN: 1311-8080 (printed version); ISSN: 1314-3395 (on-line version)

url: http://www.ijpam.eu

doi: http://dx.doi.org/10.12732/ijpam.v86i1.6

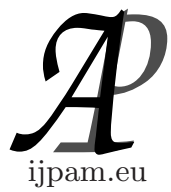

\title{
NUMERICAL APPROXIMATION OF HEAT EQUATION USING HAAR WAVELETS
}

\author{
Sharanjeet Dhawan ${ }^{1}$, Sangeeta Arora ${ }^{2}$, Sheo Kumar ${ }^{3} \S$ \\ ${ }^{1,3}$ Department Of Mathematics \\ Dr. B.R. Ambedkar National Institute of Technology \\ Jalandhar, INDIA \\ ${ }^{2}$ Department of Computer Science and IT \\ HMV College Jalandhar, INDIA
}

\begin{abstract}
This paper starts with a basic introduction to Haar wavelets which have been used for numerical simulation of heat equation. Wavelet collocation scheme is applied for the problems. Numerical experiments are carried out for some test problems. High accuracy of the results even in the case of a small number of collocation points is observed.
\end{abstract}

Key Words: Haar wavelets, numerical simulation

\section{Introduction}

Differential equations have several applications in several fields such as: physics, fluid dynamic and geophysics etc. However it is not always possible to get the solution in closed form and thus, numerical methods come into the picture. There are several numerical methods to handle a variety of problems: Finite Difference Method, Spectral Method, Finite Element Method, Finite Volume Method and so on. Many researchers are involved in developing various numerical schemes for finding solutions of different problems (see eg. [1, 2]). In this paper we consider one dimensional time-dependent heat conduction equation

Received: March 1, 2013

(C) 2013 Academic Publications, Ltd.

$\S_{\text {Correspondence author }}$ 


$$
\rho c \frac{\partial \theta(x, t)}{\partial t}=\kappa \frac{\partial^{2} \theta(x, t)}{\partial x^{2}}, \quad 0<x<L
$$

with given conditions $\theta(x, 0)=f, \theta(0, t)=g_{1}, \theta(L, t)=g_{2}$, where $\rho, c, \kappa, f, g_{1}, g_{2}$ are known functions. Problems involving heat conduction appear in many areas of engineering and science. Equation (1) appears in many mathematical models of engineering and science. So, finding out flexible techniques for generating the solutions of such PDEs is quite meaningful. Across two layer slabs, an analogy for one dimensional transient heat conduction and solution is proposed in [3] by using method of separation of variables. To solve the similar one dimensional transient heat conduction in a composite slab with layers in perfect thermal contact, is developed by [4] using the method of separation of variables, transient response of the multilayered composite conducting slabs to sudden variation of temperature of the surrounding fluid is being analyzed. Wei [5] has considered one dimensional heat equation to determine a moving boundary from cauchy data. Many researchers has used different techniques to solve the above problem and similar ones $[6,7]$.

From the past few years, wavelets have become very popular in the field of numerical approximations $[8,9]$. Among the different wavelet families mathematically most simple are the Haar wavelets. Due to the simplicity the Haar wavelets are very effective for solving ordinary differential and partial differential equations. In the previous years, many researchers have worked with Haar wavelets and their applications $[10,11]$. In order to take the advantages of the local property, many authors researched the Haar wavelet to solve the differential and integral equations [12]-[15]. In the present work, we use haar wavelets for solving heat conduction problem numerically. Numerical example shows the results obtained and comparison made with the available solution.

\section{Description of Solution Scheme}

Haar wavelet is one of the oldest and simplest wavelet. Therefore, any discussion of wavelets starts with the Haar wavelet. Due to the simplicity the Haar wavelets are very effective for solving ordinary differential and differential equations. Haar functions have been used from 1910 when they were introduced by the Hungarian mathematician Alfred Haar [10, 11, 12]. The first curve $h_{0}(t)$ also known as scaling function is defined as

$$
h_{0}= \begin{cases}1, & 0 \leq x<1 \\ 0, & \text { otherwise }\end{cases}
$$


and second curve $h_{1}$ is obtained after distributing the interval $[0,1]$ in $[0,0.5]$ and $[0.5,1]$.

$$
h_{1}=\left\{\begin{aligned}
1, & 0 \leq x<\frac{1}{2} \\
-1, & \frac{1}{2} \leq x<1
\end{aligned}\right.
$$

This is also called mother wavelet. All other subsequent curves are generated from $h_{1}(t) . h_{2}(t)$ is obtained from $h_{1}(t)$ with dilation. Other way, we can express haar functions in a more compact form as

$$
h_{n}(x)=h_{1}\left(2^{j} x-k\right), n=2^{j}+k, j \geq 0,0<k \leq 2^{j} .
$$

Having benefits of the Haar wavelet approach for its simplicity and sparse matrices of presentation, they are faster than others. Any function which is square integrable in the interval $[0,1)$, can be expanded in a Haar series with an infinite number of terms as

$$
u(x)=\sum_{i=0}^{\infty} \alpha_{i} h_{i}(x), i=2^{j}+k, j \geq 0,0 \leq k \leq 2^{j}, x \in[0,1),
$$

where Haar coefficients $\alpha_{i}=2^{j} \int_{0}^{1} u(x) h_{i}(x) d x$ are determined in such a way that the integral square error

$$
E=\int_{0}^{1}\left[u(x)-\sum_{i=0}^{m-1} \alpha_{i} h_{i}(x)\right],
$$

is minimum where $m=2^{j}, j \in\{0\} \cup N$. In general, for the function $u(x)$ to be smooth the series (4) contains an infinite number of terms. If $u(x)$ is a piecewise constant or may be approximated as piecewise constants, then the sum in equation (5) will be terminated after $m$ terms, that is

$$
u(x)=\sum_{i=0}^{m-1} \alpha_{i} h_{i}(x),
$$

where $t \in[0,1)$ and $\alpha_{m}=\left[\alpha_{0}, \alpha_{1}, \ldots, \alpha_{m-1}\right]^{T}$. we have haar functions as

$$
\begin{aligned}
h_{0} & =\left[\begin{array}{llll}
1 & 1 & 1 & 1
\end{array}\right], \\
h_{1} & =\left[\begin{array}{llll}
1 & 1 & -1 & -1
\end{array}\right], \\
h_{2} & =\left[\begin{array}{llll}
1 & -1 & 0 & 0
\end{array}\right], \\
h_{3} & =\left[\begin{array}{llll}
0 & 0 & 1 & -1
\end{array}\right],
\end{aligned}
$$


Identifying the collocation points as $x_{l}=\frac{2 l-1}{2 m} ; l=1,2, \ldots m$, we have

$$
\begin{aligned}
H_{m \times m} & =\left[h_{m}\left(x_{0}\right), h_{m}\left(x_{1}\right), \ldots, h_{m}\left(x_{m-1}\right)\right], \text { and } \\
H_{m \times m}^{-1} & =\frac{1}{m} H_{m \times m}^{T} \operatorname{diag}(r), \text { where } \\
r & =\left[11224444 \ldots \frac{m}{2}, \frac{m}{2}, \frac{m}{2}, \ldots, \frac{m}{2}\right],
\end{aligned}
$$

for $m>2$. In other way the coefficients matrix $H_{i l}=h_{i}\left(x_{l}\right)$ is introduced which is expanded into Haar series with coefficient matrix $P$ as

$$
\int_{0}^{1} h_{m}(x) d x \cong P_{m \times m} h_{m}(x), t \in[0,1) .
$$

where $m \times m$ square matrix $P$ is called the operational matrix of integration and cam be expressed as

$$
P_{m}=\frac{1}{2 m}\left(\begin{array}{cc}
2 m \mathbf{P}_{m / 2} & -\mathbf{H}_{m / 2} \\
\mathbf{H}_{m / 2}^{-1} & \mathbf{O}_{m / 2}
\end{array}\right),
$$

in particular, we get $P_{1 \times 1}=[1 / 2]$ and $\mathbf{O}_{m / 2}$ is null matrix of order $m / 2 \times m / 2$. The other elements of the matrices $H, P$ can be evaluated as

$$
\begin{aligned}
H_{2} & =\left(\begin{array}{rr}
1 & 1 \\
1 & -1
\end{array}\right), P_{2}=\frac{1}{4}\left(\begin{array}{rr}
2 & -1 \\
1 & 0
\end{array}\right) \\
H_{4} & =\left(\begin{array}{rrrr}
1 & 1 & 1 & 1 \\
1 & 1 & -1 & -1 \\
1 & -1 & 0 & 0 \\
0 & 0 & 1 & -1
\end{array}\right), P_{4}=\frac{1}{16}\left(\begin{array}{rrrrr}
8 & -4 & -2 & -2 \\
4 & 0 & -2 & 2 \\
1 & 1 & 0 & 0 \\
1 & -1 & 0 & 0
\end{array}\right) \\
H_{8} & =\left(\begin{array}{rrrrrrrrr}
1 & 1 & 1 & 1 & 1 & 1 & 1 & 1 & \\
1 & 1 & 1 & 1 & -1 & -1 & -1 & -1 \\
1 & 1 & -1 & -1 & -1 & -1 & -1 & -1 \\
0 & 0 & 0 & 0 & 1 & 1 & -1 & -1 \\
1 & -1 & 0 & 0 & 0 & 0 & 0 & 0 \\
0 & 0 & 1 & -1 & 0 & 0 & 0 & 0 \\
0 & 0 & 1 & -1 & 0 & 0 & 0 & 0 \\
0 & 0 & 0 & 0 & 0 & 0 & 1 & -1
\end{array}\right)
\end{aligned}
$$




$$
P_{8}=\frac{1}{64}\left(\begin{array}{rrrrrrrr}
32 & -16 & -8 & -8 & -4 & -4 & -4 & -4 \\
16 & 0 & -8 & 8 & -4 & -4 & 4 & 4 \\
4 & 4 & 0 & 0 & -4 & 4 & 0 & 0 \\
4 & 4 & 0 & 0 & -4 & 4 & 0 & 0 \\
1 & 1 & 2 & 0 & 0 & 0 & 0 & 0 \\
1 & 1 & -2 & 0 & 0 & 0 & 0 & 0 \\
1 & -1 & 0 & 2 & 0 & 0 & 0 & 0 \\
1 & -1 & 0 & -2 & 0 & 0 & 0 & 0
\end{array}\right)
$$

In the next section, we use these haar matrices to approximate the solution to the governing differential equation. For the governing differential equation, $x \in[a, b]$, the interval is partitioned into $2 M$ subintervals of equal length. Dividing the given interval into $N$ equal parts of length $\Delta t=(0,1] / N$ and denote defining $t_{s}=(s-1) \Delta t, s=1,2, \ldots, N$. We use the approximation

$$
\ddot{\theta}^{\prime \prime}(x, t)=\sum_{i=0}^{m-1} \alpha_{i} h_{i}(x),
$$

where $h_{i}(x)$ are haar wavelet functions and $\alpha_{i}$ are wavelet coefficients. From (12), we have

$$
\begin{aligned}
& \theta^{\prime \prime}(x, t)=\left(t-t_{s}\right) \sum_{i=0}^{m-1} \alpha_{i} h_{i}(x)+u^{\prime \prime}\left(x, t_{s}\right), \\
& \dot{\theta}(x, t)=\sum_{i=0}^{m-1} \alpha_{i} p_{i, 2}(x)+x \dot{u}^{\prime}(0, t)+\dot{u}(0, t) .
\end{aligned}
$$

Using given boundary conditions, $(13,14)$ are to be replaced in the governing equation in hand and set of equations so obtained is solved for each step.

\section{Test Problems}

In this section some test examples are analyzed to demonstrate the effectiveness of proposed method of solution. For the first case we consider the given heat equation (1) representing an iron bar of length $50 \mathrm{~cm}$. For numerical simulation, we have $f=5-\frac{1}{5}|x-25|, g_{1}=g_{2}=0,0 \leq x \leq 50$. With specific heat $c=0.437 \mathrm{~J} /(\mathrm{gK})$, density $\rho=7.88 \mathrm{~g} / \mathrm{cm}^{3}$ and thermal conductivity $\kappa=0.836 \mathrm{~W} /(\mathrm{cmK})$. Results so obtained are compared with the exact solutions. Analytical results and solution profiles obtained by the present technique 
are shown in fig. 1. Patterns of the profiles so obtained can be visualized in three dimension in fig. 2. In the second case, we consider the governing heat equation with $f=100 \sin \pi x, 0 \leq x \leq L, t>0$. Graphical results are
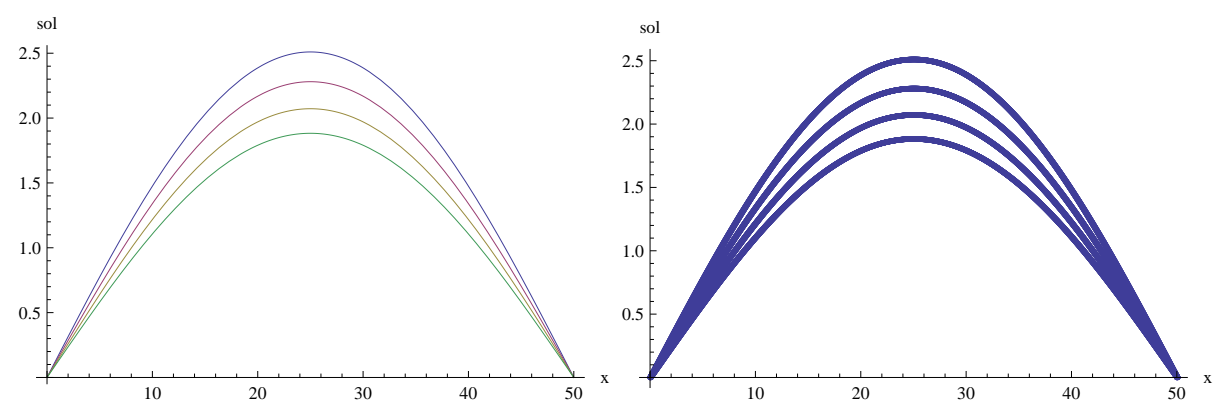

Figure 1: Exact solution and numerical solutions for first case study with $\rho=7.88 \mathrm{~g} / \mathrm{cm}^{3}, c=0.437 \mathrm{~J} /(\mathrm{gK}), \kappa=0.836 \mathrm{~W} /(\mathrm{cmK})$ at $t=$ $500,600,700,800$ respectively.

shown in Fig. (1) results are presented for $\alpha=1, t=0$ and $t=0.5$ respectively. In Fig. (2), Physical behaviour of the solution profile can be seen for $\alpha=0.5$ and $\alpha=0.01$ respectively. Results are found in good agreement with the exact solutions. Graphical representation of the numerical results can be seen in fig. 3 for $t=0.1,0.2,0.3,0.4$. Corresponding three dimensional plots are available in fig. 4. In the third example, we have rod of length $L=1$, and $k=0.1$. With initial temperature distribution, $f=0$, and the given boundary conditions $g_{1}=g_{2}=0$ and results are shown in fig. 5 .
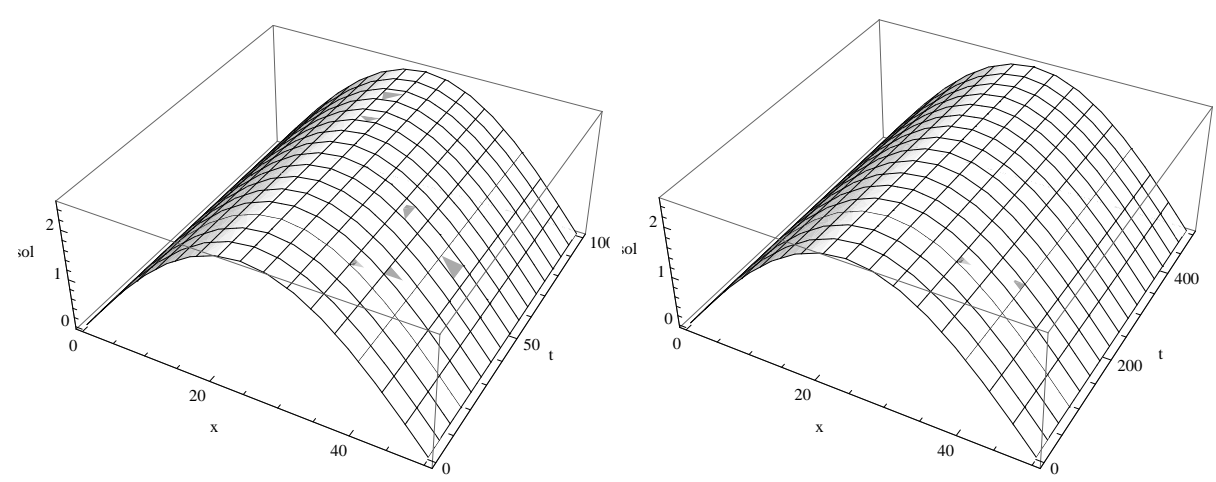

Figure 2: Physical behaviour of the solution profiles for first case in three dimension. 

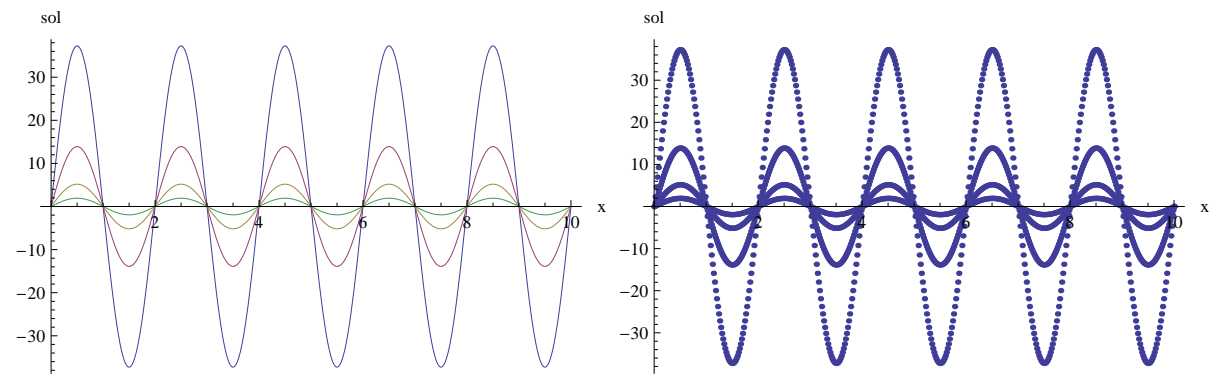

Figure 3: Solution profiles obtained for second case at $t=$ $0.1,0.2,0.3,0.4$ respectively.
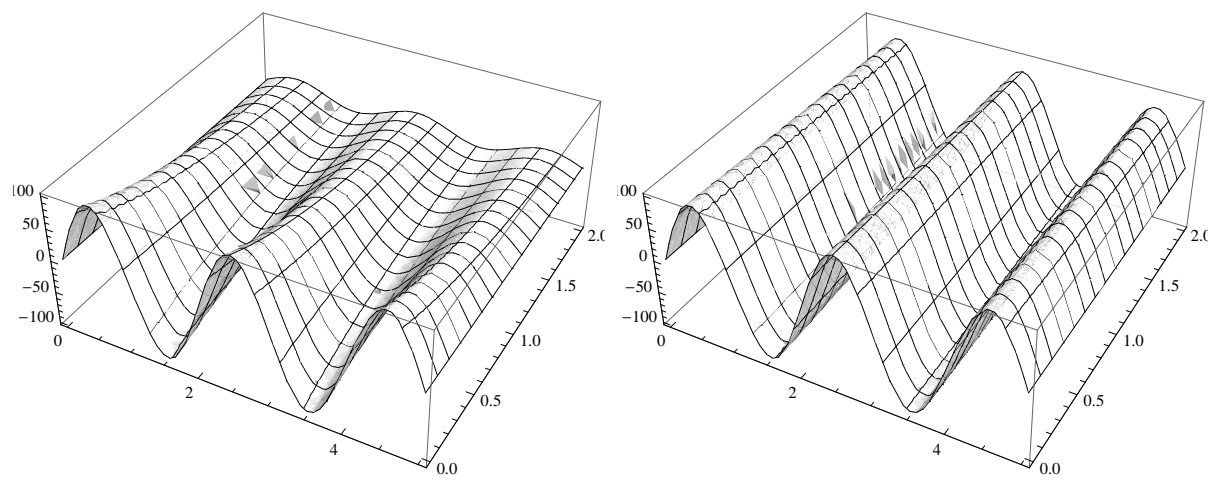

Figure 4: Physical behaviour of the solution profiles for second case in three dimension.

\section{Acknowledgments}

Financial support (No-2/40(11)/2012-R \& D-II/6575) from NBHM, DAE, Govt. of India is gratefully acknowledged. 

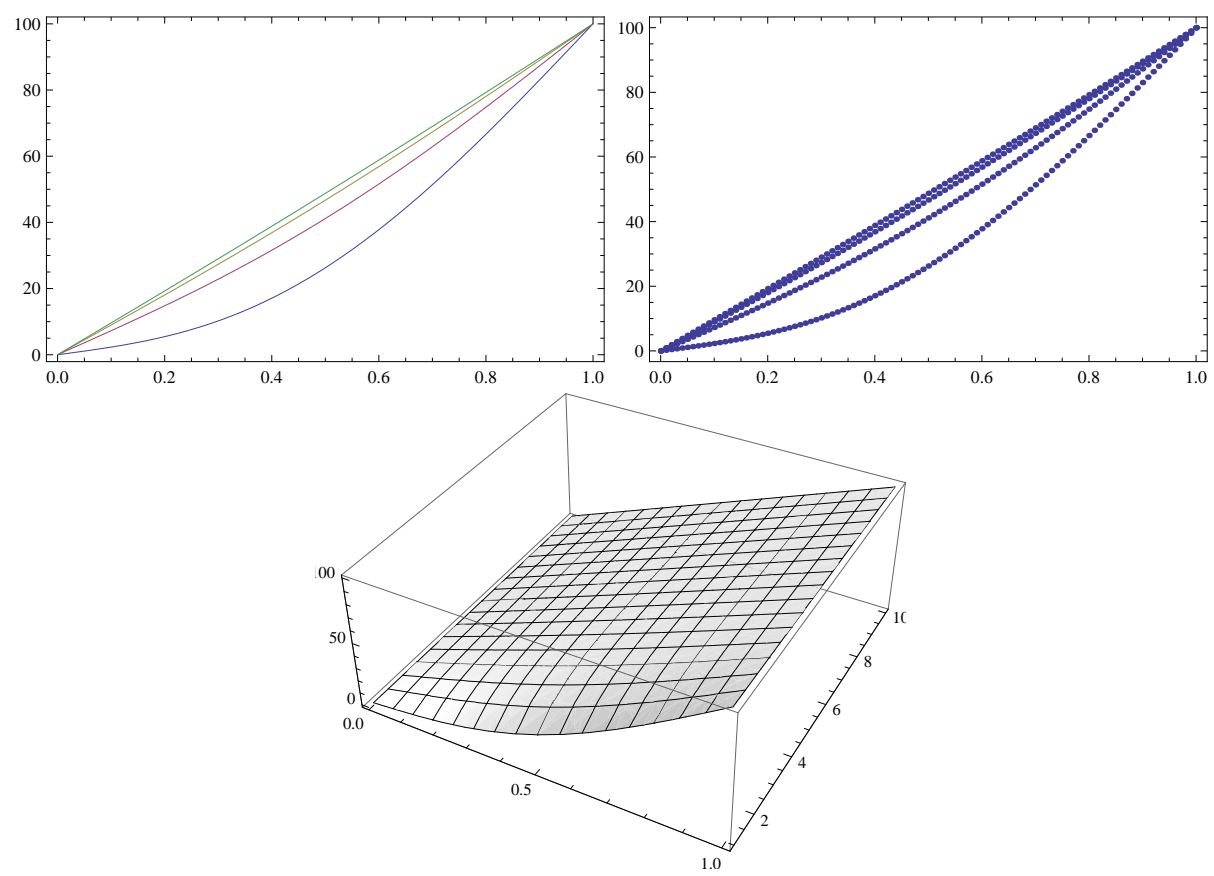

Figure 5: Solution profiles for the third case.

\section{References}

[1] P. Singh, Kapil K. Sharma, Numerical approximations to the transport equation arising in neuronal variability, International Journal of Pure and Applied Mathematics, 69 (2011), 341-356.

[2] M.K. Kadalbajoo, A. Awasthi, A numerical method based on cranknicolson scheme for Burgers' equation, Appl. Math. Comput., 182 (2006), 1430-1442.

[3] Giuseppe Pontrelli, Filippo de Monte, Mass diffusion through two-layer media: An application to the drug-eluting stent, International Journal of Heat and Mass Transfer, 50 (2007), 3658-3669.

[4] F. de Monte, Transient heat conduction in one-dimensional composite slab: A 'natural' analytic approach, International Journal of Heat and Mass Transfer, 43 (2000), 3607-3619.

[5] T. Wei, M. Yamamoto, Reconstruction of Moving Boundary from Cauchy Data in One Dimensional Heat Equation, UTMS (2008). 
[6] R.I. Medvedskii, Yu.A. Sigunov, Method of Numerical solution of onedimensional multifront stefan problems, Inzhenerno-Fizicheskii Zhurnal, 58 (1989), 681-689.

[7] L. Jones Tarcius Doss, A.K. Pani, S. Padhy, Galerkin method for a Stefantype problem in one space dimension, Numerical Solution for Partial Differential Equations, 13, No. 4 (1998), 393-416.

[8] I. Daubechies, Ten Lectures on Wavelets, SIAM, Philadelphia, PA (1992).

[9] G. Kaiser, A Friendly Guide to Wavelets, Boston, Birkhauser (1994).

[10] C.F. Chen, C.H. Hsiao, Haar wavelet method for solving lumped and distributedparameter systems, IEE Proc., No. 144 in Control Theory Appl. (1997), 87-94.

[11] C.H. Hsiao, State analysis of linear time delayed systems via Haar wavelets, Mathematics and Computers in Simulation, 44 (1997), 457-470.

[12] U. Lepik, Numerical solution of evolution equations by the Haar wavelet method, Appl. Math. and Comput., 185 (2007), 695-704.

[13] U. Lepik, Numerical solution of differential equations using Haar wavelets, Mathematics and Computers in Simulation, 68 (2005), 127-143.

[14] F. Khellat, S.A. Yousefi, The linear Legendre mother wavelets operational matrix of integration and its application, J. Franklin Inst., 143 (2006), 181-190.

[15] J.-Y. Xiao, L.-H. Wen, D. Zhang, Solving second kind Fredholm integral equation by periodic wavelet Galerkin method, Appl. Math. Comput., 175 (2006), 508-518. 
